\title{
Spin-to-orbital conversion of the angular momentum of light and its classical and quantum applications
}

Lorenzo Marrucci, Ebrahim Karimi, Sergei Slussarenko, Bruno Piccirillo, Enrico Santamato, Eleonora Nagali, and Fabio Sciarrino

J. Opt. 13, 064001 (2011)

\section{Copyright 2011 - Institute of Physics Publishing Ltd}

This is an author-created, un-copyedited version of an article accepted for publication in the Journal of Optics. IOP Publishing Ltd is not responsible for any errors or omissions in this version of the manuscript or any version derived from it. The Version of Record is available online at http://dx.doi.org/10.1088/2040-8978/13/6/064001 


\title{
Spin-to-orbital conversion of the angular momentum of light and its classical and quantum applications
}

\author{
Lorenzo Marrucci, ${ }^{1,2}$ Ebrahim Karimi, ${ }^{1}$ Sergei Slussarenko, ${ }^{1}$ Bruno \\ Piccirillo, ${ }^{1,3}$ Enrico Santamato, ${ }^{1,3}$ Eleonora Nagali, ${ }^{4}$ and Fabio Sciarrino ${ }^{4,5}$ \\ ${ }^{1}$ Dipartimento di Scienze Fisiche, Università di Napoli "Federico II", \\ Complesso di Monte S. Angelo, 80126 Napoli, Italy \\ ${ }^{2}$ CNR-SPIN, Complesso di Monte S. Angelo, 80126 Napoli, Italy* \\ ${ }^{3}$ Consorzio Nazionale Interuniversitario per le Scienze Fisiche della Materia, Napoli, Italy \\ ${ }^{4}$ Sapienza Università di Roma, Roma, Italy \\ ${ }^{5}$ Consiglio Nazionale delle Ricerche - Istituto Nazionale di Ottica, Italy
}

\begin{abstract}
Few years ago the possibility of coupling and inter-converting the spin and orbital angular momentum (SAM and OAM) of paraxial light beams in inhomogeneous anisotropic media was demonstrated. An important case is provided by wave-plates having a singular transverse pattern of the birefringent optical axis, with a topological singularity of charge $q$ at the plate center, hence named "q-plates". The introduction of q-plates has given rise in a few years to a number of new results and to a significant progress in the field of orbital angular momentum of light. Particularly promising are the quantum photonic applications, because the polarization control of OAM allows the transfer of quantum information from the SAM qubit space to an OAM subspace of a photon and vice versa. In this paper, we review the development of the q-plate idea and some of the most significant results that have originated from it, and we will briefly touch on many other related findings concerning the interaction of the SAM and OAM of light.
\end{abstract}

\section{INTRODUCTION: SPIN-TO-ORBITAL ANGULAR MOMENTUM CONVERSION}

The formal separation of the angular momentum of an optical field into a spin part (SAM) and an orbital part (OAM) was first proposed by J. Humblet in 1943 [1]. Although a number of papers have been published on this topic since that first work (see, e.g., [2] and references therein), the problem of introducing a physically unambiguous separation of SAM and OAM of arbitrary optical fields remains still controversial and debated (see, e.g., [3-8]). Nevertheless, such a separation becomes clearly unambiguous and physically meaningful in the paraxial limit [9]. A paraxial beam has a well defined SAM that is associated with its circular polarization content. The OAM, however, can be further split into two components [10]: (i) an external one, that arises from the cross product of the total momentum transported by the beam and the position of its axis relative to the origin of coordinates; (ii) an internal OAM component that is associated with the helical structure of the optical wavefront around the beam axis and with an optical vortex located at the beam axis [9]. The internal OAM acts as a sort of additional spin of the whole beam around its axis, as it is always oriented parallel to the beam axis and it is independent of the choice of the origin of coordinates. However, while the SAM per photon can only take two values, namely $S_{z}= \pm \hbar$, where $\hbar$ is the reduced Planck constant and $z$ is the beam axis, the OAM per photon can be any positive or negative integer multiple of $\hbar$, i.e. $L_{z}=m \hbar$ with $m$ any integer. The integer $m$ also defines the phase variation of the optical beam seen when circling its axis, i.e., the wavefront phase factor $\exp (\operatorname{im} \varphi)$ where $\varphi$ is the azimuthal angle around the optical axis $z$. The spin and orbital angular momentum components can be also distinguished according to their different mechanical action on small absorbing particles, with the SAM inducing spin of the particle independent of its position, while the OAM induces revolution of the particle around the beam axis [10-12]. The possibility of distinguishing between SAM and OAM according to their coupling with different rotational degrees of freedom of optical media has been also considered [13].

Since SAM is associated with optical polarization and OAM with optical wavefront, at first sight they appear to be quite separate and non-interacting properties of light, at least in the paraxial limit. For this reason, for about ten years since the publication of the seminal paper by L. Allen et al. which started the current field of research in the optical OAM, the possibility of an interaction between SAM and internal OAM taking place in a single paraxial optical beam was not considered. The generation and control of optical OAM has been based only on essentially polarization-independent tools, such as cylindrical lenses [9], spiral phase plates [14], holograms [15-17] (including reconfigurable ones made using spatial light modulators), and Dove prisms, also in suitable interferometric setups [18, 19].

In 2002, following an original idea by R. Bhandari [20], the group of E. Hasman in Technion University reported the use of patterned subwavelength diffraction gratings for reshaping the wavefront of an electromagnetic wave, so as to obtain various wavefronts, including helical beams $[21,22]$. The underlying concept was that of manipulating the beam polarization so as to introduce a space-variant Pancharatnam-Berry phase. For this rea- 
son, these phase devices were called Pancharatnam-Berry optical elements (PBOE). Subwavelength grating PBOEs were however experimentally demonstrated only for midinfrared electromagnetic waves, due to manufacturing limitations (subwavelength-grating PBOEs working in the near-infrared have been reported only recently [23]). In 2006, L. Marrucci et al. (initially unaware of Hasman's work) proposed that anisotropic inhomogeneous media such as the liquid crystals could give rise to a previously unrecognized optical process in which the variation of SAM occurring from the medium birefringence gives rise to the appearance of OAM, arising from the medium inhomogeneity [24]. In rotationally symmetric geometries, this process involves no net transfer of angular momentum to matter, so that the SAM variation in the light is entirely converted into its OAM. For this reason, the process was dubbed "spin-to-orbital conversion of angular momentum" (STOC). Marrucci and coworkers demonstrated also experimentally this process with visible light, by using patterned liquid crystal cells that were called "q-plates" [24, 25]. The first demonstration of the STOC process for single photons and photon pairs was reported a few years later by F. Sciarrino and coworkers [26].

This advance did not arise out of the blue. The Naples optics group had been working on the exchange of angular momentum of light with anisotropic media such as liquid crystals (including puzzling enhancement effects arising when the liquid crystals are doped with certain dyes) since the mid eighties (see, e.g., [13, 27-32]). The STOC process idea actually sprung from the observation that radially oriented liquid crystal droplets trapped into circularly polarized laser beams did not rotate [33] and from the ensuing question, asked by I. Jánossy, about the fate of the spin optical angular momentum in such a process. The conceptual answer provided by the STOC idea has been subsequently directly demonstrated in a later experiment performed just with liquid crystal droplets [34]. A detailed analysis of the torques and forces arising in the liquid crystal droplet by effect of the interaction with light has been later reported by Jánossy [35].

The q-plate was first conceived as a "simplified" (flattened) liquid crystal droplet. In a more general definition, a q-plate is a slab of a birefringent material, e.g., a liquid crystal, having a uniform birefringent phase retardation $\delta$ and a transverse optical axis pattern with a nonzero topological charge. The pattern is defined by the number $q$ of rotations that the optical axis exhibits in a path circling once around the center of the plate, where a topological defect must be present ( $q$ is negative if the direction of the axis rotation is opposite to that of the path). The number $q$ must clearly be integer or semi-integer, since the optical axis has no polarity. $q$ is also the charge of the topological singularity located at the center of the plate. A secondary parameter defining the q-plate pattern is the initial optical axis orientation, as for example specified by the angle $\alpha_{0}$ it forms at a reference angular position in the transverse plane (e.g., on the $x$ axis in the plate). Some examples of q-plate patterns are shown in Fig. 1.
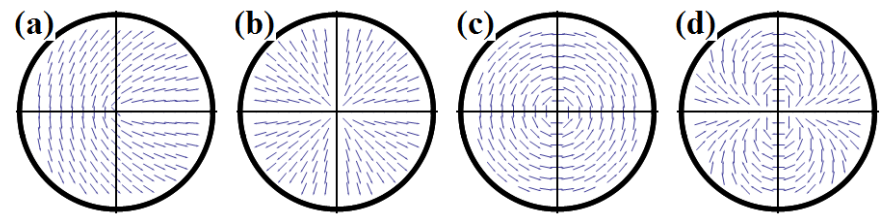

FIG. 1. Four examples of q-plate patterns. (a) $\left(q, \alpha_{0}\right)=$ $\left(\frac{1}{2}, 0\right),(\mathrm{b})\left(q, \alpha_{0}\right)=(1,0)$, (c) $\left(q, \alpha_{0}\right)=\left(1, \frac{\pi}{2}\right)$ and (d) $\left(q, \alpha_{0}\right)=(2,0)$. The segments indicate the optical axis orientation in the transverse plane.

For $\delta=\pi$, i.e. at so-called optimal tuning, a q-plate modifies the OAM state $m$ of a circularly polarized light beam passing through it, imposing a variation $\Delta m= \pm 2 q$ whose sign depends on the input polarization, positive for left-circular and negative for right-circular. The handedness of the output circular polarization is also inverted, i.e. the SAM is flipped. In other words, a q-plate gives rise to a polarization-controlled variation of OAM. In particular, in the rotationally symmetric case $q=1$, the OAM variation balances the SAM one, so that the total angular momentum of the light beam is conserved, and we have a pure STOC process. This behavior is pictorially illustrated in Fig. 2. An untuned q-plate with $\delta \neq \pi$
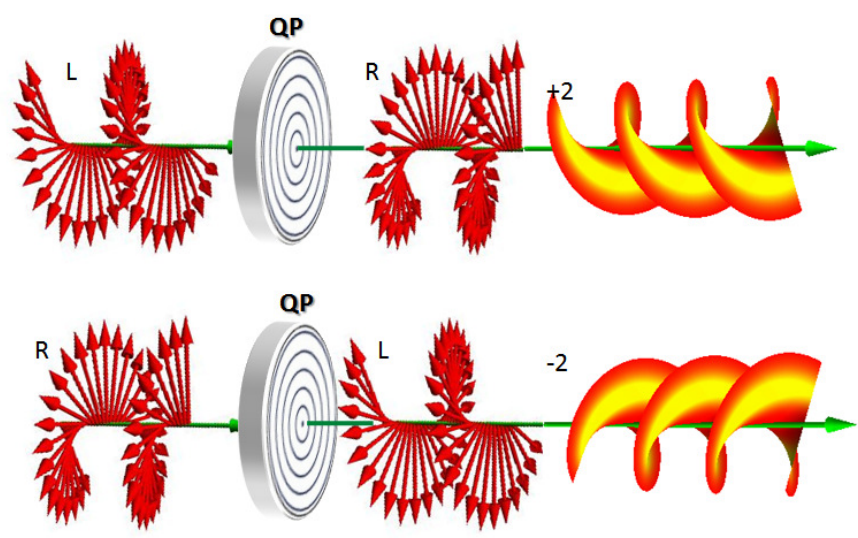

FIG. 2. Pictorial illustration of the optical action of a tuned q-plate on a input circularly-polarized plane-wave light, for the case $q=1$. The output is a helical mode with OAM given by $m= \pm 2$, with the sign determined by the input polarization handedness.

will give rise to a superposition of a wave transformed just as for the tuned case and an unmodified wave, with amplitudes respectively given by $\sin (\delta / 2)$ and $\cos (\delta / 2)$. These optical properties of the q-plate can be simply derived using the Jones matrix calculus, valid in the limit of negligible transverse diffraction inside the q-plate (i.e., 
very thin q-plates) [36].

A tuned q-plate allows one to generate a pure helical beam carrying nonzero OAM (with $m= \pm 2 q$ ) starting with a circularly polarized gaussian input mode (having $m=0$ ), with very high efficiency (ideally close to $100 \%$ ), no deflection of the propagation axis and with a polarization-controlled handedness [37, 38]. q-plates can therefore provide a very convenient approach to generating OAM beams, which can compete with computergenerated holograms and spatial light modulators. The polarization control of the OAM sign allows high-speed switching with rates that in principle can reach $\mathrm{GHz}$ rates [25]. Even more interestingly, the polarization control of the OAM sign allows developing new kinds of quantum manipulations of single photons, as has been demonstrated in a series of experiments mainly performed by F. Sciarrino and coworkers in Roma's quantum optics group, which will be reviewed below. In particular, as we will show, the combined use of polarization and OAM for accessing a high-dimensional quantum space attached to each photon is progressively enabling the implementation of novel promising quantum information protocols $[39,40]$.

Inhomogeneous birefringent media such as the q-plates are not the only systems in which STOC can take place. A inhomogeneous dichroic medium, such as a spacevariant polarizer with a q-plate-like optical axis geometry, can give rise to very similar phenomena (with the advantage of an achromatic response and the disadvantage of significant optical losses) [41]. An electro-optical device allowing a polarization-controlled OAM manipulation quite similar to the q-plate one, based on a pair of opposite spiral phase plates having electrically controlled refractive index, has been theoretically proposed recently [42]. A STOC phenomenon bearing many similarities to that taking place in a q-plate with $q=1$ may also occur in a homogeneous uniaxial birefringent crystal, when an optical beam propagates along the optical axis of the crystal. This was first proved theoretically by A. Ciattoni et al. [43, 44] and experimentally by E. Brasselet et al. [45-47]. A similar phenomenon has been shown to occur in a biaxial crystal by internal conical diffraction $[48,49]$. In contrast to the case of q-plates, however, this approach is limited to generating OAM $m= \pm 2$, due to the rotational symmetry of the medium. Moreover, the conversion efficiency in the paraxial limit cannot be higher than 50\%. Another interesting situation in which a form of STOC takes place is when an initially paraxial circularly-polarized beam passes through a short-focal lens. The resulting strongly-focused non-paraxial beam exhibits an OAM content, as demonstrated experimentally by particle manipulation experiments $[50,51]$. In this case, however, the OAM per photon remains small and its effects are clearly visible only close to the beam focus. The possibility of an electro-optical modulation of this effect has also been reported [52]. Another recent work showed that optical beams having a radially varying SAM also acquire an additional rather unexpected component of OAM-like angular momentum, presumably arising as a consequence of departure from the paraxial limit [53].

Moreover, the interaction of SAM and external OAM, that is at the basis of the so-called optical spin Hall effect, has also been recently conceived and experimentally demonstrated $[54,55]$. Related spin-orbit optical phenomena are the polarization "geometrodynamics" $[56,57]$ and the polarization-based optical sensing of nano-particle displacements [58]. It should be furthermore mentioned that several works in the field of singular optics [59], that is strictly related with that of OAM, have recently tackled issues concerning the interaction between polarization and wavefront structures in the optical field (see, e.g., [60-62]). Finally, an emerging field in which the spin-orbit interaction of SAM and OAM may bear fruitful results in the near future is that of optical polariton condensates in semiconductor microcavities (see, e.g., $[63,64]$ ).

In the following of this article, we discuss some of the main developments that have arisen since the first introduction of the q-plate and the observation of the STOC process. The article is organized as follows. In Section II we survey the developments in the technology for manufacturing and tuning the liquid crystal q-plates and mention some nonlinear phenomena in which a q-plate-like geometry takes place spontaneously. Section III concerns the theory of optical propagation inside a q-plate and the resulting optical modes at the q-plate output. Section IV is mainly about the optical setups for OAM manipulation that can be obtained by combining one or more q-plates in suitable optical schemes, but it includes a brief survey of related results of polarization-based OAM manipulation. Quantum applications of the q-plates and of SAMOAM photon interactions are finally discussed in Section V.

\section{Q-PLATES MANUFACTURING AND TUNING}

The main issue to be addressed in the manufacturing of q-plates is the patterning of the optical axis. Liquid crystals (LC) are soft birefringent materials allowing flexible spatial patterning of the average molecular orientation that defines the optical axis. LCs can be aligned by several methods. For static alignment, the simplest choice is to use the so-called "surface anchoring", i.e., a treatment of the bounding substrates that generates a preferential molecular alignment of the LC in contact with the surface. For dynamical alignment one can use external fields, such as magnetic, electric or even optical fields. LC q-plates can be then manufactured as thin (order of $5-10 \mu \mathrm{m}$ ) LC films, sandwiched between two glass substrates which have been previously coated with a suit- 
able alignment layer, typically made of polymer, such as polyimide or other materials. These materials are suitable for aligning the LC optical axis parallel (or slightly tilted) to the bounding surfaces, i.e. the so-called "planar anchoring". To single out a specific direction in the plane one can then use a mechanical rubbing procedure (using velvet or other fabrics) of the polymer-coated substrate. It is however hard to introduce an arbitrary pattern by mechanical rubbing and this approach is convenient only in the case of the the simplest geometry corresponding to $q=1$, which is rotationally symmetric. For this practical reason all the early experimental works with q-plates were using q-plates with $q=1$.

A more versatile and clean approach to patterning LC cells is to use a photoinduced alignment method of the polymer coating of the containing substrates, as proposed in [25]. In this approach, the anisotropy of the polymer is controlled by the linear polarization of the writing light, which defines the material optical axis (either parallel or perpendicular to the writing field polarization). There are different permanent orienting effects of light on the polymer coatings which can be used. The most common ones are either photochemical, i.e. based on selectively destroying or creating chemical links by preferential absorption, or photophysical, i.e. based on the photoinduced selective reorientation of dye molecules dispersed in the polymer. One can use this approach to directly write an anisotropic pattern in a thin polymer film that becomes itself a q-plate, as for example recently reported in Ref. [65]. However, all polymer q-plates are not dynamically tunable, as their birefringent retardation $\delta$ is fixed by the film thickness and by the polymer degree of alignment and corresponding birefringence. We instead recently demonstrated the photoinduced alignment approach to prepare patterned polymer-coated substrates with which we could assemble tunable patterned LC qplates with arbitrary topological charge $q$ [66]. In figure 3 a LC q-plate manufactured by the photoalignment method is shown, together with the interference patterns demonstrating the helical structure of the outgoing wavefront.

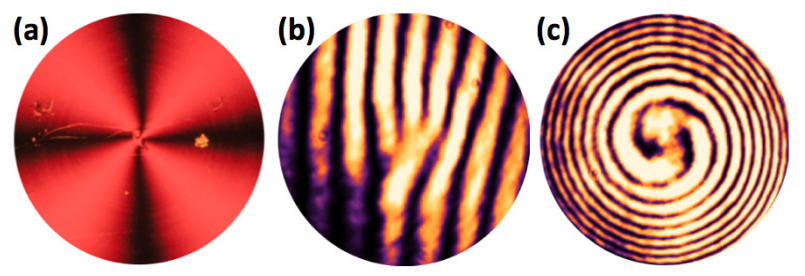

FIG. 3. (a) a $q=1$ q-plate prepared by a photoalignment technique, as seen between crossed polarizers. (b-c) Interference patterns of the outgoing beam from the q-plate with (b) planar and (c) spherical reference waves, for a left-circular input polarization.

The tuning of a LC q-plate, that is controlling the bire- fringence phase retardation $\delta$, useful for optimizing the STOC process or to adjust it for different wavelengths can be achieved by different methods, including mechanical pressure, thermal methods, and external-field induced LC reorientation. So far, a thermal approach exploiting the strong dependence of the LC birefringence on temperature [38] and an electric one, exploiting the electric-field induced reorientation of the LC molecular alignment [67] have been demonstrated. The latter of course allows for a relatively fast dynamical control of tuning, while the former is more suitable for static tuning.

Since the STOC process is accompanied by polarization helicity inversion, in the case of pure circularly polarized input beam the STOC and non-STOC components of the output light can be simply separated by a polarizing beam-splitter (PBS), because the converted and non-converted light will have orthogonal polarization states. This allows for a very simple measurement of the STOC efficiency and of the phase retardation $\delta$ that controls it, as shown for example in Fig. 4. In this

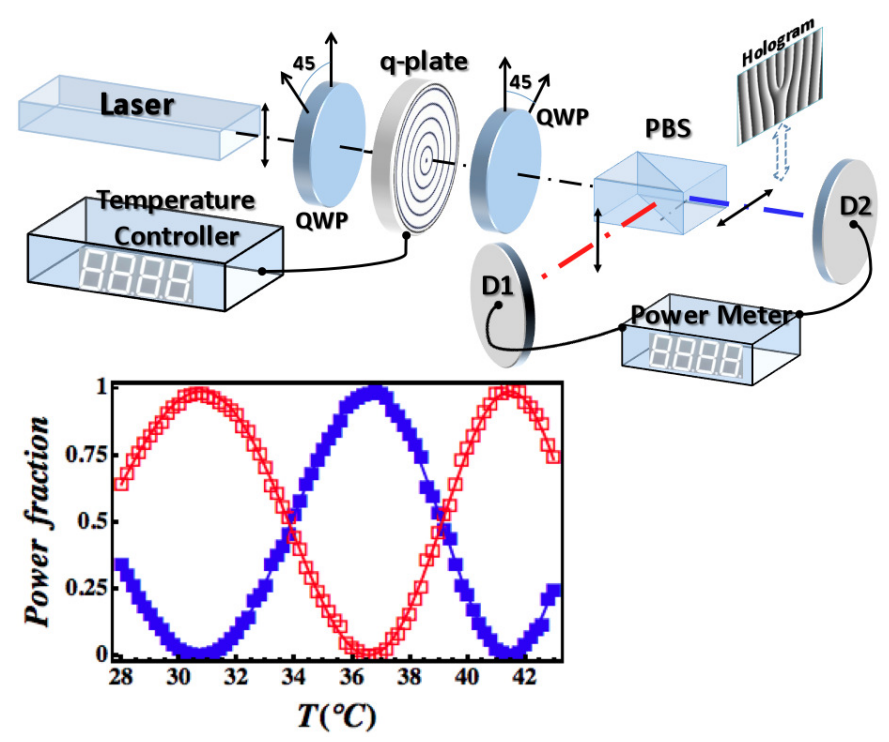

FIG. 4. Setup used to measure the STOC efficiency and the state purity. Legend: QWP-quarter wave plate, PBSpolarizing beam-splitter. The fork hologram was inserted on the converted beam arm for verifying the degree of purity of the OAM $m=2$ mode generated on the output. STOC power fraction (blue $\mathbf{\square}$ ) and no STOC power fraction (red $\square)$ as functions of the $q$-late temperature. The curves are theoretical best fits. [38].

experiment, the optimal STOC efficiency exceeded $99 \%$, neglecting reflection and diffraction losses (85\% taking into account losses, which however were not minimized with anti-reflection coatings).

Before concluding this Section, we should mention that certain light-induced modifications of materials, for example associated with thermal-induced stresses [68, 69], as well as nonlinear optical phenomena such as those 
taking place in LCs themselves in suitable geometries $[70,71]$, have been shown to be capable of spontaneously generating q-plate-like structured media, thus giving rise to STOC phenomena, although usually not with very high conversion efficiency. In such cases, the STOC concept provides anyway a convenient framework for revealing and analyzing the associated optical effects.

\section{OPTICAL PROPAGATION THROUGH Q-PLATES AND OUTGOING MODES}

The simplest theory of optical propagation through the q-plate is based on the assumption that at each transverse position $x, y$ the propagation is independent (as for a local plane wave), which corresponds to the geometrical optical approximation. The only effect of the birefringence is then that of altering the polarization and introducing a Pancharatnam-Berry phase, as can be derived using a simple Jones matrix approach. This simple theory, proposed in the early papers $[24,25,36,72]$, neglects any transverse diffraction effects arising in the propagation. This is certainly a very good approximation in the limit of thin q-plates and wide beams, but not applicable for points that are very close to the central singularity, where the rapid transverse spatial variations associated with the singularity are expected to give rise to significant diffraction effects.

To go beyond the geometric optical approximation, one needs to model the diffraction of the helical waves traveling in the q-plate and emerging from it. Being helical, such modes are often simply referred to as LaguerreGauss (LG) modes. However, while the azimuthal phase factor of the helical modes is always just the same as for a pure LG mode, their radial structure may be different, and in general one can only say that helical modes can be written as superpositions of LG modes having different radial index $p$ and a same azimuthal index $m$. It can be shown that for an ideally thin q-plate, as well as for any spiral phase plate, the outgoing modes obtained in the paraxial limit when at input there is a pure $\mathrm{TEM}_{00}$ gaussian mode, form a subset of a general class of modes that has been recently introduced, the "hypergeometric gaussian modes" [73].

These same modes are also useful for describing the propagation inside the q-plate. An approximate analytical solution of this problem, exploiting the q-plate symmetry in the limit of high beam paraxiality, was proposed in Ref. [37]. The radial profile of the optical beam at any transverse plane $z$ inside the q-plate (or at the output face) is found to be independent of the cumulated birefringent optical retardation at $z$ and can be given in terms of superposition of two ordinary and extraordinary hypergeometric-gaussian modes. As a consequence, the intensity patterns of the outgoing beam of a tuned or detuned q-plate in the near field are identical. Nevertheless, in the far field, the gaussian or doughnut shapes of the intensity profile will reemerge depending on the OAM, as it is shown in Fig. (5).
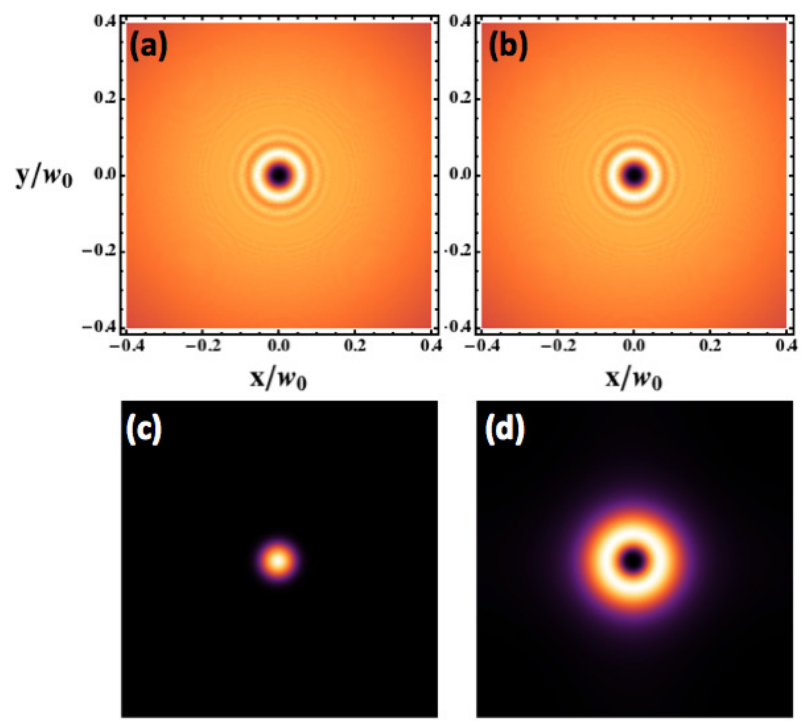

FIG. 5. Intensity profile at the exit face of the $q=1$ qplate. (a) No SAM-to-OAM conversion; (b) Full SAM-toOAM conversion. Intensity profile in the far-field beyond the q-plate. (c) No SAM-to-OAM conversion; (d) Full SAM-toOAM conversion. [37]. A circularly polarized $\mathrm{TEM}_{00}$ input beam has been assumed.

The cumulated optical retardation of the plate controls the SAM-to-OAM conversion, or STOC, as shown in Fig. 6. Compared with the geometric-optical approximation, this more exact theory predicts a very slow decrease of the optimal efficiency with increasing q-plate thickness, due to internal diffraction.

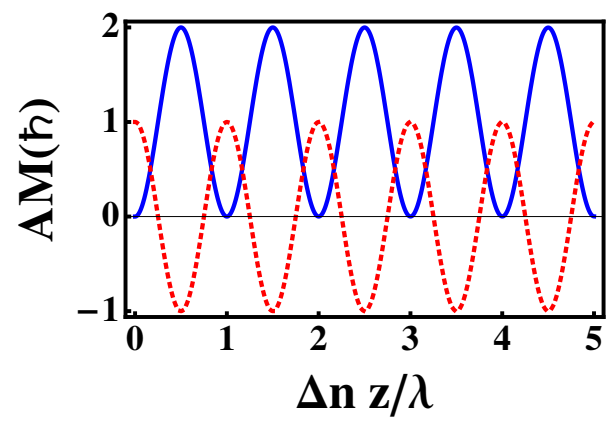

FIG. 6. OAM (blue solid line) and SAM (red dotted line) as function of the optical retardation $\Delta n z / \lambda$ for thin $q=1$ q-plate. The input beam is left-circularly polarized TEM 00 . We have assumed the following data: LC refractive ordinary and extraordinary indices $n_{o}=1.5$ and $n_{e}=1.7$, respectively, and beam waist $w_{0}=50 \lambda$.

An exact vectorial theory of rotationally symmetric Bessel beams propagating in q-plates with $q=1$ and of 
the associated STOC phenomenon has been also reported [74]. An interesting analogy with the Aharonov-Bohm effect for the propagation of light in a nematic liquid crystal with a disclination, corresponding to a q-plate geometry, has been proposed by A. M. de M. Carvalho et al. [75].

\section{MANIPULATION OF AZIMUTHAL MODES VIA POLARIZATION}

The q-plate and the resulting SAM-OAM coupling and STOC process have provided the basis for several novel optical devices and setups for manipulating the OAM of light. Many of these devices, although they are essentially classical, find a natural important application in the quantum information setting, so we will come back to them in the following section.

An important concept is the one-to-one mapping that can be established between the space $\pi$ of all possible (generally elliptical) polarization states and an arbitrary two-dimensional subspace of the OAM degree of freedom, spanned by two opposite OAM values $m= \pm \ell$, hereafter denoted as $o_{\ell}$. This subspace is defined independently of the radial mode (this is for example appropriate if the radial mode is separable and can be factorized). Both $\pi$ and $o_{\ell}$ spaces are two-dimensional complex vector spaces having the same structure as the Hilbert space of a quantum spin, or as the representations of the $\mathrm{SU}(2)$ group. Neglecting a global phase, any point in such a space can be represented as a point on a three-dimensional sphere, the Poincaré sphere in the case of polarization, or an analogous sphere in the OAM case [76] (both being analogous to Bloch's sphere used for quantum spins). An arbitrary state in an OAM $o_{\ell}$ subspace is generally not a pure helical beam, but it can be always written as the superposition of the two opposite OAM basis states. This is just the same as for the polarizations, which in general are not associated with well defined values of the photon SAM, but can be always decomposed into a superposition of the two circularly polarized waves with opposite handedness.

Now, a q-plate in combination with other optical devices allows one to physically implement just this mapping. There are both unitary and non-unitary schemes. The non-unitary schemes waste a fraction of the input optical energy (i.e., they are "probabilistic", in the language of quantum information, because a fraction of the photons are lost). However they can be very simple. For example a single q-plate followed by a linear polarizer allows one to transfer the polarization input state into the corresponding OAM subspace $o_{\ell}$ with $\ell=2 q$ [77]. This scheme however has a $50 \%$ efficiency (or success probability). The opposite transfer, from OAM to SAM, can be obtained by combining a q-plate with a single-mode fiber, used for filtering $m=0$ states [77]. This is again a scheme with $50 \%$ efficiency.
A $100 \%$ efficient mapping, that is a unitary scheme, can be obtained by combining a q-plate and one ore two Dove prisms inserted into an interferometer, such as a Mach-Zehnder or (more conveniently) a Sagnac [77, 78]. This scheme, illustrated in Fig. 7, is fully reciprocal and can therefore work in both directions (see also Ref. [79] for another proposed optical scheme, not based on the q-plate, in which the OAM state is controlled by polarization via a Sagnac interferometer). This scheme was

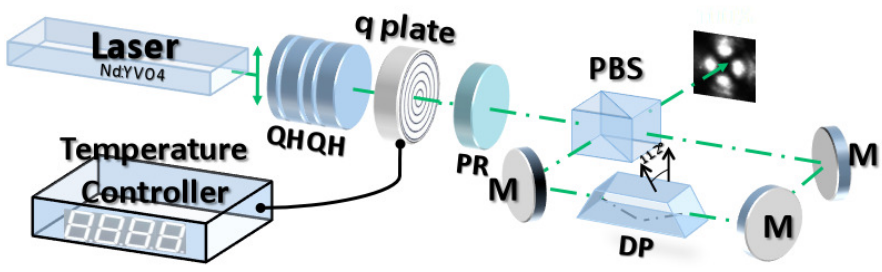

FIG. 7. Scheme of an experimental setup based on a qplate and a Dove prism in a polarizing Sagnac interferometer, that allows for 100\%-efficient transfer of an arbitrary SAMencoded input state into a OAM bi-dimentional state or vice versa. Legend: $\mathrm{QHQH}$ - set of wave-plates to generate an arbitrary polarization state; PR - polarization rotator; PBS polarizing beam-splitter; DP - Dove prism; M- mirror [78].

demonstrated experimentally in the classical regime [78]. An additional feature of this setup is that also the geometrical Pancharatnam-Berry phase arising from the polarization manipulations is transferred to the OAM output beam. Examples of the resulting modes on the OAM Poincaré sphere for $\ell=2$ are given in Fig. 8. The interference fringes with a reference beam, also shown, were used to analyze the output mode phase structure and to measure the geometric phase.
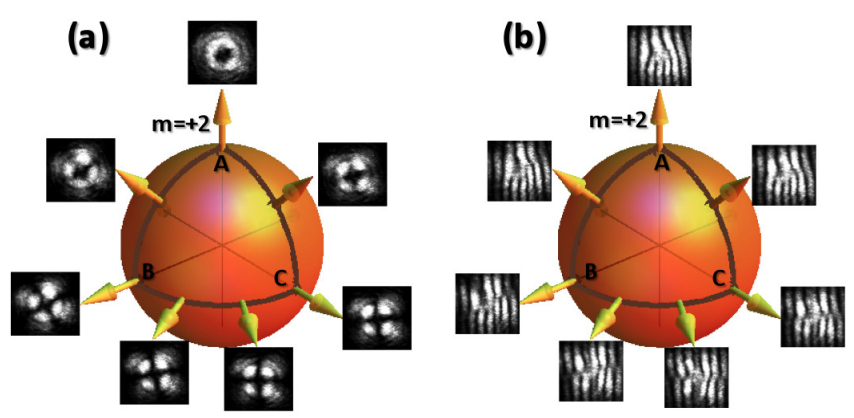

FIG. 8. A possible closed path over the OAM-Poincaré sphere. The path starts and ends at the pole. (a) Intensity profiles of the generated beam at different points of the path. (b) Corresponding interference patterns with a $\mathrm{TEM}_{00}$ linearly-polarized reference beam [78].

It must be emphasized that this setup can generate a class of azimuthal transverse modes, including all modes that have the same azimuthal structure as the HermiteGaussian modes, with a theoretical efficiency of $100 \%$. 
The choice of the generated mode is entirely controlled by the input polarization, which can be manipulated at very fast rates. This should be contrasted with the more limited efficiency of the spatial light modulators (typically below $70 \%$ ) and slow response (less than $1 \mathrm{kHz}$ ). The possibility of controlling even a four-dimensional OAM subspace, including both $m= \pm 2$ and $m= \pm 4$ states, by a single q-plate inserted in a optical loop scheme has been also reported very recently [80].

A different optical scheme, still based on the q-plate, can be used as a spin-orbit SAM-OAM four-dimensional mode sorter and detector [38]. The four-dimensional space is the tensor product of the polarization space $\pi$ and a OAM subspace $o_{\ell}$. In other words, arbitrary optical states are defined as linear combinations of four basis states $|L, \ell\rangle,|L,-\ell\rangle,|R, \ell\rangle$ and $|R,-\ell\rangle$, where $L, R$ denote the left and right circular polarizations. The sorting is based on two steps. First, a q-plate-induced shifting of the OAM value of the beam. Assuming that the input beam has an OAM given by the number $m=\ell$ (in units of $\hbar$ ), the q-plate will convert it into either $m=0$ or $m=2 \ell$, depending on the input polarization handedness. This requires using a q-plate with $q=\ell / 2$ (in the reported experiment, $q=1$ and $\ell=2$ were used). Next, the beam is split according to the outgoing circular polarization and further separated by radial sectioning, e.g. by using a mirror with a hole to reflect only the external doughnut component and let the central spot pass. This radial sectioning exploits the coupling between the OAM and the radial profile that emerges during free propagation. The small residual overlap of the two radial modes however gives rise to a non-perfect contrast ratio. The contrast ratio can be improved without limitations at the expense of detection efficiency by blocking the annular region where mode overlap occurs.

The last device that we mention in this Section was proposed to perform arbitrary unitary transformations in the spin-orbit four-dimensional space $\pi \otimes o_{\ell}$, keeping always the single-beam structure (i.e., without splitting the beam into an interferometer scheme) [81]. It is based on a complex combination of q-plates, birefringent waveplates and lenses, and it again exploits the coupling between radial mode and OAM arising in the free propagation. This setup was proposed mainly with quantum applications in mind, as it provides a universal quantum gate for the spin-orbit Hilbert space of a single photon. But being a single photon device it can be also discussed as a classical optics device, so we discuss it here.

The working principle of this device is similar to that of the spin-orbit mode sorter just described, as it exploits the spatial separation of the $m=0$ and $m=2 \ell$ modes occurring in the radial coordinate in the free propagation. An important element is the so-called "q-box" (QB), which is made of two q-plates and a unitary polarization gate sandwiched between them (which is essentially a combination of suitable wave plates and isotropic phase retardation plates). The radius of the wave plates of the spin gate is selected so to act only on the $m=0$ mode, leaving the $m=2 \ell$ one unchanged. The propagationinduced coupling between the OAM and the radial coordinate is controlled by suitable lenses to switch between near-field and far-field and back. A schematic illustration of the q-box device is given in Fig. 9. A sequence of four

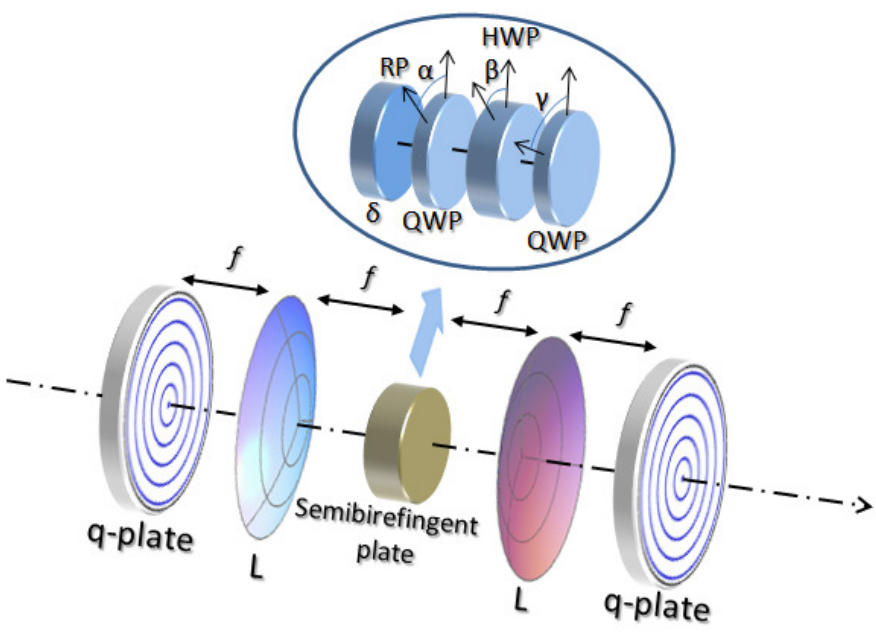

FIG. 9. A scheme of a q-box. A SAM (polarization) unitary gate is shown in the inset [81]. Legend: QWP-quarter wave plate, HWP-half wave plate, L-lens, RP-retardation plate.

q-boxes separated by quarter and half wave plates (QWP and $\mathrm{HWP}$ ) in the following order: $\mathrm{QB} \rightarrow \mathrm{QWP} \rightarrow \mathrm{QB} \rightarrow$ $\mathrm{HWP} \rightarrow \mathrm{QB} \rightarrow \mathrm{QWP} \rightarrow \mathrm{QB}$ will make a 16-parameter unitary gate that will correspond to a $4 \times 4$ unitary matrix which is universal, meaning that by adjusting the parameters one can realize any unitary operation on the spin-orbit optical state. Such highly complex setup, however, is not always necessary. Many important gates can be realized with much fewer elements. For example, the CNOT gate can be realized with a single q-box having a single half wave plate inside.

Because of the presence of residual overlaps of $m=0$ and $m=2 \ell$ modes in the radial coordinate, the fidelity of the q-box is not $100 \%$. An optimal selection of the radius of the wave-plates in the q-box can provide a minimum fidelity of about $83 \%$ for $\ell=2$ (but the fidelity increases if higher order OAM modes are considered and it can become close to $100 \%$ for specific input-output states). As for the mode sorter, the fidelity can be increased at the expense of efficiency (success probability in the quantum applications) by stopping the annular regions where the overlap takes place. 


\section{QUANTUM INFORMATION APPLICATIONS: QUBITS AND QUDITS IN SPIN-ORBIT STATES}

The interest of the OAM degree of freedom of light in the quantum information field [82] mainly arises from the possibility of using its high-dimensionality for encoding a large amount of information in single photons and from the robustness of such encoded information due to the angular momentum conservation law $[2,83]$. The standard unit of quantum information is the qubit, a twodimensional quantum state. OAM allows the encoding of qubits by exploiting any two-dimensional subspace, such as the $o_{\ell}$ ones or others. However, many quantum information protocols benefit from the use of so-called "qudits", that are higher-dimensional quantum states, for encoding the information. OAM provides an obvious possibility for qudit optical implementation, by exploiting a larger subspace. Moreover, OAM can be readily combined with other degrees of freedom of the photon in order to further expand the Hilbert space or to realize the so-called hyper-entanglement, where different degrees of freedom of two particles are simultaneously entangled $[84,85]$. In particular, the combination of OAM with SAM is of special interest here, given the similar nature of the two degrees of freedom and given the possibility, offered by devices such as the q-plate, of coupling and manipulating the two degrees of freedom together. Another interesting potential application of combining SAM and OAM is to create a frame-invariant encoding of quantum information [86, 87].

The action of a q-plate on a single photon quantum state is essentially the same as for the fields of classical coherent light. Let us introduce the ket notation $|P, m\rangle=|P\rangle_{\pi}|m\rangle_{o}$ for the single photon states, where $P$ stands for the polarization state (e.g., $P=L, R, H, V$ for left and right circular polarizations and horizontal and vertical linear polarizations) and $m$ is the OAM value in units of $\hbar$. The radial state is omitted for brevity (this is possible when the radial state can be factorized out). The q-plate action (here, and in the following, optimal tuning is assumed), which can be associated to a quantum evolution operator $\widehat{\mathrm{QP}}$, is then described by the following rules:

$$
\begin{aligned}
& \widehat{\mathrm{QP}}|L\rangle_{\pi}|m\rangle_{o}=|R\rangle_{\pi}|m+2 q\rangle_{o} \\
& \widehat{\mathrm{QP}}|R\rangle_{\pi}|m\rangle_{o}=|L\rangle_{\pi}|m-2 q\rangle_{o}
\end{aligned}
$$

When applied to an input linearly polarized light (e.g., horizontal) having $m=0$, we obtain the following output state:

$$
\widehat{\mathrm{QP}}|H\rangle_{\pi}|0\rangle_{o}=\frac{1}{\sqrt{2}}\left(|L\rangle_{\pi}|-2 q\rangle_{o}+|R\rangle_{\pi}|2 q\rangle_{o}\right)
$$

The right-hand-side expression can be interpreted as an entangled state of the SAM (or polarization) and OAM degrees of freedom of the same photon (of course this kind of single-particle entanglement does not involve nonlocality effects). These predictions have been tested experimentally on heralded single photon states obtained by parametric parametric down-conversion (SPDC) [26].

An interesting application of the q-plate is for realizing optical devices that can transfer the quantum information initially stored in the polarization degree of freedom of the photon into the OAM degree of freedom, or vice versa. In other words, these devices may implement the following transformations (in both directions):

$$
|\psi\rangle_{\pi}|0\rangle_{o} \leftrightarrows|H\rangle_{\pi}|\psi\rangle_{o}
$$

where $|\psi\rangle$ here stands for an arbitrary qubit state and we have chosen to use $|H\rangle_{\pi}$ as "blank" state of polarization and $|0\rangle_{o}$ as blank state of OAM (other choices are obviously possible). These quantum information transfer devices can be implemented either probabilistically or deterministically, by adopting the optical schemes presented in $[26,77,88]$ and experimentally verified in the simpler probabilistic schemes within the heralded single photon regime. These experiments showed a quantum fidelity of $97 \%$ or higher. Multiple SAM $\rightarrow$ OAM $\rightarrow$ SAM and cascaded SAM $\rightarrow$ OAM $m=|2| \rightarrow$ OAM $m=|4|$ transfers were also demonstrated [77].

The single photon manipulations discussed above, although performed in the heralded mode ensuring the presence of one and only one photon at the time, are not truly different from classical optics experiments (such as those discussed in the previous Section). Uniquely quantum effects that cannot be explained with classical theories only arise when dealing with more than one particle. The generation of a biphoton state with nontrivial OAM quantum correlations was demonstrated in Ref. [26], again using a q-plate. The experiment is illustrated in Fig. 10. A biphoton state is a single optical

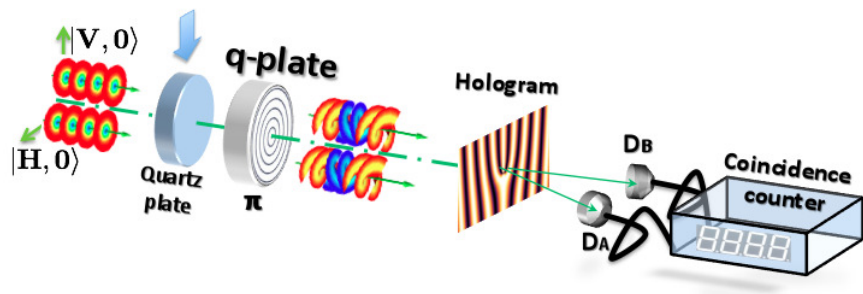

FIG. 10. The schematic of biphoton OAM coalescence setup. The SPDC source generates pairs of correlated photons having $H$ and $V$ polarizations. The q-plate converts this correlation in the OAM space. The correlations can then be tested by the vanishing coincidence measurements on opposite OAM states, as filtered using a fork hologram. A quartz plate can be used to introduce a delay between the two input photons, thus destroying the quantum correlations. [26].

mode having exactly two photons. In the reported experiment, a biphoton state having polarization correlations 
was initially generated by SPDC, and the SAM-OAM transfer device was then used to generate the final state with OAM correlations. It must be noted that the qplate acted on the two photons simultaneously in this experiment. Such action cannot be described in classical terms. After erasing the polarization degree of freedom, the biphoton state finally generated can be described as follows [26]:

$$
\frac{1}{\sqrt{2}}\left(|+2\rangle_{o}|+2\rangle_{o}+|-2\rangle_{o}|-2\rangle_{o}\right)
$$

where the OAM values were in the subspace with $\ell=2$ because the employed q-plate had $q=1$. The successful generation of this state can be verified by testing for OAM correlations occurring when detecting two-photon coincidences. In particular, a vanishing number of counts is expected when testing for coincidences between opposite values of the OAM. The coincidence counts are instead restored if the two photons are delayed, e.g. by inserting a suitable birefringent crystal such as a quartz plate, so as to become distinguishable. A typical "coincidence dip" behavior is therefore observed, as shown in Fig. 11. Other tests on the biphoton state, including

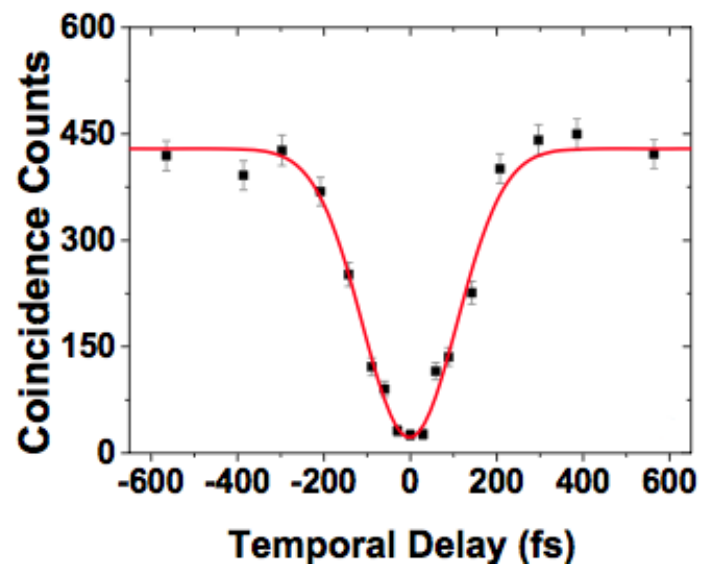

FIG. 11. Coincidence counts obtained in the measurement of opposite OAM values for the biphoton generated by the q-plate, as illustrated in Fig. (10)

a verification of the enhanced coalescence probability in OAM and a test of coherence based on analyzing the two photons in OAM-superposition states by suitable holograms have also been performed. We refer the readers to Ref. [26] for further details.

Thanks to the SAM-OAM quantum transfer devices, an arbitrary qubit photon state can be initially prepared in the SAM space and then transferred into the OAM space. Any OAM state can be also conveniently analyzed by simply transferring it back to the SAM space. This makes the OAM utilization in quantum photonic experiments much easier than it was before. This technology step has for example allowed the first experimental demonstration of Hong-Ou-Mandel (HOM) coalescence
[89] of OAM-carrying photons in a beam splitter [90]. This phenomenon results from the two-photon interference between the photons impinging on a beam-splitter from two different input ports. Such interference, due to the bosonic nature of photons, leads to a doubled probability for the two photons to emerge together from the same output port of the beam-splitter and a vanishing probability for the two photons to emerge from the two separate output ports. This works only when the two photons are indistinguishable, i.e. the impinging wavepackets are synchronous and the SAM and OAM states of the two photons are the same (after taking into account the reflection inside the beam-splitter). This behavior was well demonstrated in the experiment [90]. The importance of this proof-of-principle demonstration is that the HOM coalescence effect is an enabling process, on which many other quantum information protocols are based (such as the quantum teleportation, quantum cloning, etc.). And indeed, in the same paper the implementation of optimal quantum cloning of OAMencoded photonic qubits was also demonstrated [90].

The transfer devices have also recently enabled the preparation of spin-orbit hybrid-entangled photons [91, 92]. Two opposite approaches have been demonstrated. By starting with a polarization (SAM) entangled pair generated by SPDC, one can transfer the quantum information of only a single photon of the pair from SAM to OAM to obtain the hybrid entanglement [91]. Conversely, by starting with a OAM entangled pair (also generated by SPDC, as first demonstrated in [93]), it is possible to reach the hybrid entanglement by a OAMto-SAM quantum transfer [92]. In these works, the entanglement has been confirmed by testing the violation of a Bell's inequality [91, 92, 94]. The non-separability (or single-particle entanglement) of SAM and OAM degrees of freedom has been also investigated, using q-plates or interferometric layouts, both in a classical intense beam regime $[92,95]$ and in a heralded single-photon one $[26,92,94,96]$. The remote preparation of singleparticle hybrid entangled states has been also recently demonstrated (not using q-plates), by exploiting a SAMOAM hyper-entangled photon pair source [97]. A proposal for hybrid-entanglement multi-photon manipulations exploiting the q-plate has also been recently put forward [98].

Finally, the most recent progress in the use of SAMOAM coupling for quantum information has been based on combining both degrees of freedom for encoding qudits. Even though some implementation of quantum states with dimension higher than 2 have been already carried out with biphoton states [100-103], an appealing goal is that of encoding as much information (i.e. as many qubits) as possible in a single photon by exploiting different degrees of freedom, so as to exceed the limitations due to noise interactions. In particular, by encoding a qubit in SAM and another qubit in a OAM sub- 


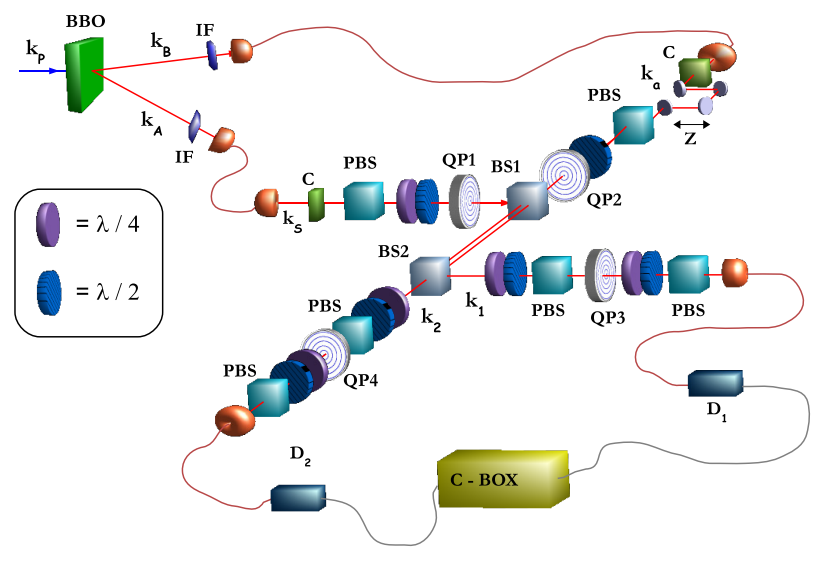

FIG. 12. Experimental apparatus for implementing the $1 \rightarrow 2$ optimal quantum cloning of polarization-OAM photon ququarts [99].

space one obtains a single-photon "ququart", i.e. a quantum state with dimension $d=4$ defined in the Hilbert space $\pi \otimes o_{l}$. The preparation and measurement of single photon ququarts by using a q-plate based apparatus was demonstrated in Ref. [104]. Interestingly, in this work all states belonging to the five mutually unbiased basis that span the four-dimensional Hilbert space have been generated and measured, including those characterized by an entanglement between OAM and polarization. The next step has then been to demonstrate a first quantum protocol on such ququarts, that is, the optimal quantum cloning $1 \rightarrow 2$ of ququarts achieved by exploiting the four-dimensional HOM effect [99], as illustrated in Fig.12.

The ability to clone a ququart codified in the OAMpolarization space in all the mutually unbiased bases has also been experimentally verified by reconstructing their density matrices through quantum state tomography. The overlap between clones and original quantum state experimentally observed is in good accordance with theoretical predictions involving the increasing dimensionality of the quantum system [90, 105].

An interesting result obtained by working within the SAM-OAM 4D space has been the experimental investigation (not based on the q-plate device) of the topological phase arising in transformations taking place in the space of maximally entangled SAM-OAM states, that provides a representation of the $\mathrm{SO}(3)$ group [106]. The SAMOAM continuous-variable hyper-entanglement has been also demonstrated recently by the same group [107].

In addition to the experimental works discussed above, a number of theoretical works have explored many other possible applications of the q-plate, or more generally of employing a SAM-OAM combination, in the quantum information field. For example, the possibility of using a q-plate for increasing the effective Shannon dimensionality of an entangled pair of photons generated by SPDC, as a consequence of spin-orbit hyper-entanglement, has been proposed [108]. Related works exploiting the combination of SAM and transverse-mode degrees-of-freedom entanglement in SPDC have also been reported, showing also a link with the field of quantum imaging [109]. A three-degree-of-freedom single-photon entanglement, involving SAM, OAM, and optical frequency, can be generated by means of rotating q-plates, thanks to a rotational doppler shift effect [110]. The implementation of so-called quantum walks in OAM space, or in hybrid SAM-OAM space, has been also investigated very recently $[111,112]$.

We notice that several single-photon quantum information protocols involving SAM-OAM combined manipulations can be also implemented without making recourse to q-plates, by using suitable interferometric layouts in combination with OAM manipulating devices such as spiral phase plates, Dove prisms, cylindrical lenses, etc. (see, e.g., $[95,113,114])$. In all these setups, the main working principle is the conversion of the polarization qubit into a path (or "dual rail") qubit and vice versa, by means of polarizing beam splitters. Although these approaches are fully appropriate for demonstration purposes, an obvious practical advantage of the q-plate-based setups is that they do not require interferometric stability and may often carry out the entire desired quantum manipulation remaining within a single beam geometry.

\section{CONCLUSIONS}

In summary, we have surveyed recent progress in the field of the orbital angular momentum of light, with specific attention to its interaction with the optical spin angular momentum, i.e. the light polarization. The first reported experimental demonstrations of the possibility of inter-converting the SAM and OAM of a paraxial light beam and of single photons have stimulated an intense research effort in the last few years. In particular, new methods of generation, control, and manipulation of the optical OAM, both in the classical and quantum regimes, have been demonstrated using the recently introduced device named q-plate. Such device is relatively easy to manufacture, it is tunable and highly efficient. Its action introduces a controlled coupling between polarization (SAM) and OAM that can be conveniently exploited in many different ways. The combination of one or more q-plates and more standard polarization or OAM devices (such as the Dove prism) in suitable schemes allows achieving many new optical functionalities. In addition, a number of schemes and experiments not using the qplate, but still exploiting the SAM and OAM of light and their reciprocal interaction, have been recently reported.

Perhaps the most interesting potential of the combined 
use of polarization and OAM of light lies in the quantum information field, since the multi-dimensionality of the OAM space provides a natural possibility for implementing qudits within single photons. The hunt for a higher quantum dimensionality of the photon is hence open!

\section{ACKNOWLEDGMENTS}

We acknowledge the financial support of the Future and Emerging Technologies (FET) programme within the Seventh Framework Programme for Research of the European Commission, under FET-Open grant number 255914 - PHORBITECH.

\section{REFERENCES}

* lorenzo.marrucci@na.infn.it

[1] J. Humblet. Sur le moment d'impulsion d'une onde electromagnetique. Physica, 10:585-603, 1943.

[2] S. Franke-Arnold, L. Allen, and M. J. Padgett. Advances in optical angular momentum. Laser Photonics Rev., 2(4):299-313, 2008.

[3] S. J. Van Enk and G. Nienhuis. Spin and orbital angular momentum of photons. Europhys. Lett., 25(7):497-501, 1994.

[4] S. J. Van Enk and G. Nienhuis. Commutation rules and eigenvalues of spin and orbital angular momentum of radiation fields. J. Mod. Opt., 41:963-977, 1994.

[5] S. M. Barnett. Optical angular-momentum flux. J. Opt. B-Quantum Semicl. Opt., 4(2):S7-S16, 2002.

[6] T. A. Nieminen, A. B. Stilgoe, N. R. Heckenberg, and H. Rubinsztein-Dunlop. Angular momentum of a strongly focused gaussian beam. J. Opt. A-Pure Appl. Opt., 10(11), 2008.

[7] Chun-Fang Li. Spin and orbital angular momentum of a class of nonparaxial light beams having a globally defined polarization. Phys. Rev. A, 80:063814, 2009.

[8] S. M. Barnett. Rotation of electromagnetic fields and the nature of optical angular momentum. J. Mod. Optics, 57(14-15):1339-1343, 2010.

[9] L. Allen, M. W. Beijersbergen, R. J. C. Spreeuw, and J. P. Woerdman. Orbital angular momentum of light and the transformation of laguerre-gaussian laser modes. Phys. Rev. A, 45:8185-8189, 1992.

[10] A. T. O'Neil, I. MacVicar, L. Allen, and M. J. Padgett. Intrinsic and extrinsic nature of the orbital angular momentum of a light beam. Phys. Rev. Lett., 88:053601, 2002.

[11] H. He, M. E. J. Friese, N. R. Heckenberg, and H. Rubinsztein-Dunlop. Direct observation of transfer of angular momentum to absorptive particles from a laser beam with a phase singularity. Phys. Rev. Lett., 75:826829, 1995.

[12] N. B. Simpson, K. Dholakia, L. Allen, and M. Padgett. Mechanical equivalence of spin and orbital angular mo- mentum of light: an optical spanner. Opt. Lett., 22:5254, 1997.

[13] B. Piccirillo and E. Santamato. Light angular momentum flux and forces in birefringent inhomogeneous media. Phys. Rev. E, 69:056613, 2004.

[14] M. W. Beijersbergen, R. P. C. Coerwinkel, M. Kristensen, and J. P. Woerdman. Helical-wavefront laser beams produced with a spiral phaseplate. Opt. Commun., 112:321-327, 1994.

[15] V. Y. Bazhenov, M. V. Vasnetsov, and M. S. Soskin. Laser beams with screw dislocations in their wavefronts. Sov. Phys.-JETP Lett., 52:429-431, 1990. [Pis'ma Zh. Eksp. Teor. Fiz., 52, 1037-1039 (1990)].

[16] V. Y. Bazhenov, M. S. Soskin, and M. V. Vasnetsov. Screw dislocations in light wavefronts. J. Mod. Opt., 39:985, 1992.

[17] I. V. Basistiy, V. Yu. Bazhenov, M. S. Soskin, and M. V. Vasnetsov. Optics of light beams with screw dislocations. Opt. Commun., 103:422-428, 1993.

[18] J. Leach, M. J. Padgett, S. M. Barnett, S. FrankeArnold, and J. Courtial. Measuring the orbital angular momentum of a single photon. Phys. Rev. Lett., 88(25):257901, Jun 2002.

[19] S. Slussarenko, V. D'Ambrosio, B. Piccirillo, L. Marrucci, and E. Santamato. The polarizing sagnac interferometer: a tool for light orbital angular momentum sorting and spin-orbit photon processing. Opt. Express, 18:27205-27216, 2010.

[20] R. Bhandari. Polarization of light and topological phases. Phys. Rep., 281(1):1-64, 1997.

[21] G. Biener, A. Niv, V. Kleiner, and E. Hasman. Formation of helical beams by use of pancharatnam-berry phase optical elements. Opt. Lett., 27:1875-1877, 2002.

[22] Z. Bomzon, G. Biener, V. Kleiner, and E. Hasman. Opt. Lett., 27:1411, 2002.

[23] E. Lombard, A. Drezet, C. Genet, and T. W. Ebbesen. Polarization control of non-diffractive helical optical beams through subwavelength metallic apertures. New J. Phys., 12:023027, 2010.

[24] L. Marrucci, C. Manzo, and D. Paparo. Optical spinto-orbital angular momentum conversion in inhomogeneous anisotropic media. Phys. Rev. Lett., 96:163905, 2006.

[25] L. Marrucci, C. Manzo, and D. Paparo. PancharatnamBerry phase optical elements for wavefront shaping in the visible domain: switchable helical modes generation. Appl. Phys. Lett., 88:221102, 2006.

[26] E. Nagali, F. Sciarrino, F. De Martini, L. Marrucci, B. Piccirillo, E. Karimi, and E. Santamato. Quantum information transfer from spin to orbital angular momentum of photons. Phys. Rev. Lett., 103:013601, 2009.

[27] G. Abbate, P. Maddalena, L. Marrucci, L. Saetta, and E. Santamato. Photodynamical effects induced by the angular momentum of light in liquid crystals. Phys. Scripta, T39:389-393, 1991.

[28] E. Santamato, B. Daino, M. Romagnoli, M. Settembre, and Y. R. Shen. Collective rotation of molecules driven by the angular momentum of light in a nematic film. Phys. Rev. Lett., 57:2423, 1986.

[29] L. Marrucci, G. Abbate, S. Ferraiuolo, P. Maddalena, and E. Santamato. Self-induced stimulated light scattering in nematic liquid crystals: Theory and experiment. Phys. Rev. A, 46:4859-4868, 1992. 
[30] L. Marrucci and D. Paparo. Photoinduced molecular reorientation of absorbing liquid crystals. Phys. Rev. E, 56:1765-1772, 1997.

[31] B Piccirillo, C Toscano, F Vetrano, and E Santamato. Orbital and spin photon angular momentum transfer in liquid crystals. Physical Review Letters, 86(11):22852288, 2001.

[32] M. Kreuzer, E. Benkler, D. Paparo, G. Casillo, and L. Marrucci. Molecular reorientation by photoinduced modulation of rotational mobility. Phys. Rev. E, 68:011701, 2003.

[33] C Manzo, D Paparo, L Marrucci, and I Janossy. Lightinduced rotation of dye-doped liquid crystal droplets. Phys. Rev. E, 73(5, Part 1):051707, 2006.

[34] E. Brasselet, N. Murazawa, H. Misawa, and S. Juodkazis. Optical vortices from liquid crystal droplets. Phys. Rev. Lett., 103(10):103903, 2009.

[35] I. Jánossy. Electromagnetic torque and force in axially symmetric liquid-crystal droplets. Opt. Lett., 33:2371$2373,2008$.

[36] L. Marrucci. Generation of helical modes of light by spin-to-orbital angular momentum conversion in inhomogeneous liquid crystals. Mol. Cryst. Liq. Cryst., 488:148-162, 2008.

[37] E. Karimi, B. Piccirillo, L. Marrucci, and E. Santamato. Light propagation in a birefringent plate with topological unit charge. Opt. Lett., 34:1225, 2009.

[38] E. Karimi, B. Piccirillo, E. Nagali, L. Marrucci, and E. Santamato. Efficient generation and sorting of orbital angular momentum eigenmodes of light by thermally tuned q-plates. Applied Physics Letters, 94(23):231124, 2009.

[39] M. Stütz, S. Gröblacher, T. Jennewein, and A. Zeilinger. How to create and detect n-dimensional entangled photons with an active phase hologram. Appl. Phys. Lett., 90:261114, 2007.

[40] S. Straupe and S. Kulik. Quantum optics: The quest for higher dimensionality. Nat. Photon., 4:585-586, 2010.

[41] J. A. Ferrari, W. Dultz, H. Schmitzer, and E. Frins. Achromatic wavefront forming with space-variant polarizers: Application to phase singularities and light focusing. Phys. Rev. A, 76:053815, 2007.

[42] L. X. Chen and W. L. She. Electrically tunable and spindependent integer or noninteger orbital angular momentum generator. Opt. Lett., 34:178-180, 2009.

[43] A. Ciattoni, G. Cincotti, and C. Palma. Circularly polarized beams and vortex generation in uniaxial media. J. Opt. Soc. Am. A, 20:163-171, 2003.

[44] A. Ciattoni, G. Cincotti, and C. Palma. Angular momentum dynamics of a paraxial beam in a uniaxial crystal. Phys. Rev. E, 67:036618, 2003.

[45] E. Brasselet, Y. Izdebskaya, V. Shvedov, A. S. Desyatnikov, W. Krolikowski, and Y. S. Kivshar. Dynamics of optical spin-orbit coupling in uniaxial crystals. Opt. Lett., 34:1021-1023, 2009.

[46] C. Loussert and E. Brasselet. Efficient scalar and vectorial singular beam shaping using homogeneous anisotropic media. Opt. Lett., 35:7-9, 2010.

[47] T. A. Fadeyeva, V. G. Shvedov, Y. V. Izdebskaya, A. V. Volyar, E. Brasselet, D. N. Neshev, A. S. Desyatnikov, W. Krolikowski, , and Y. S. Kivshar. Spatially engineered polarization states and optical vortices in uniaxial crystals. Opt. Express, 18:10848-10863, 2010.
[48] M. V. Berry, M. R. Jeffrey, and M. Mansuripur. Orbital and spin angular momentum in conical diffraction. $J$. Opt. A, 7:685-690, 2005.

[49] D. P. ODwyer, C. F. Phelan, Y. P. Rakovich, P. R. Eastham, J. G. Lunney, and J. F. Donegan. Generation of continuously tunable fractional optical orbital angular momentum using internal conical diffraction. Opt. Express, 18:16480-16485, 2010.

[50] Y. Zhao, J. S. Edgar, G. D. M. Jeffries, D. McGloin, and D. T. Chiu. Spin-to-orbital angular momentum conversion in a strongly focused optical beam. Phys. Rev. Lett., 99:073901, 2007.

[51] H. Adachi, S. Akahoshi, and K. Miyakawa. Orbital motion of spherical microparticles trapped in diffraction patterns of circularly polarized light. Phys. Rev. A, 75:063409, 2007.

[52] L. X. Chen and W. L. She. Electro-optic ally forbidden or enhanced spin-to-orbital angular momentum conversion in a focused light beam. Opt. Lett., 33:696-698, 2008.

[53] X.-L. Wang, J. Chen, Y. Li, J. Ding, C.-S. Guo, and H.-T. Wang. Optical orbital angular momentum from the curl of polarization. Phys. Rev. Lett., 105:253602, 2010.

[54] M. Onoda, S. Murakami, and N. Nagaosa. Hall effect of light. Phys. Rev. Lett., 93(8):083901, 2004.

[55] O. Hosten and P. Kwiat. Observation of the spin hall effect of light via weak measurements. Science, 319(5864):787-790, 2008.

[56] K. Y. Bliokh, A. Niv, V. Kleiner, and E. Hasman. Geometrodynamics of spinning light. Nat. Photon., 2:748$753,2008$.

[57] K. Y. Bliokh. Geometrodynamics of polarized light: Berry phase and spin hall effect in a gradient-index medium. J. Opt. A, 11:094009, 2009.

[58] O. G. Rodríguez-Herrera, D. Lara, K. Y. Bliokh, E. A. Ostrovskaya, and C. Dainty. Optical nanoprobing via spin-orbit interaction of light. Phys. Rev. Lett., 104:253601, 2010.

[59] M. S. Soskin and M. V. Vasnetsov. Singular optics. Progress in Optics, 42:219-276, 2001.

[60] M. S. Soskin, V. Denisenko, and I. Freund. Optical polarization singularities and elliptic stationary points. Opt. Lett., 28:1475-1477, 2003.

[61] M. R. Dennis, K. O'Holleran, and M. J. Padgett. Singular optics: Optical vortices and polarization singularities. Progr. Opt., 53:293-364, 2009.

[62] I. Freund. Multitwist optical möbius strips. Opt. Lett., 35:148-150, 2010.

[63] D. Sanvitto, F. M. Marchetti, M. H. Szymanska, G. Tosi, M. Baudish, F. P. Laussy, D. N. Krizhanovskii, M. S. Skolnick, L. Marrucci, A. Lemaître, J. Bloch, C. Tejedor, and L. Vi na. Persistent currents and quantized vortices in a polariton superfluid. Nat. Phys., 6:527-533, 2010.

[64] I. A. Shelykh, A. V. Kavokin, Y. G. Rubo, T. C. H. Liew, and G. Malpuech. Polariton polarizationsensitive phenomena in planar semiconductor microcavities. Semicond. Sci. Technol., 25:013001, 2010.

[65] S. Nersisyan, N. Tabiryan, D. M. Steeves, and B. R. Kimball. Opt. Express, 17(14):11926, 2009.

[66] S. Slussarenko, A. Murauski, T. Du, V. Chigrinov, L. Marrucci, and E. Santamato. Tunable liquid crystal q-plates with arbitrary topological charge. Opt. Express, 
2011.

[67] B. Piccirillo, V. D'Ambrosio, S. Slussarenko, L. Marrucci, and E. Santamato. Photon spin-to-orbital angular momentum conversion via an electrically tunable q-plate. Appl. Phys. Lett., 97:241104, 2010.

[68] S. Mosca, B. Canuel, E. Karimi, B. Piccirillo, L. Marrucci, R. De Rosa, E. Genin, L. Milano, and E. Santamato. Photon self-induced spin-to-orbital conversion in a terbium-gallium-garnet crystal at high laser power. Phys. Rev. A, 82(4):043806, 2010.

[69] J. Morikawa, A. Orie, T. Hashimoto, and S. Juodkazis. Thermal and optical properties of the femtosecondlaser-structured and stress-induced birefringent regions in sapphire. Opt. Express, 18:8300-8310, 2010.

[70] E. Brasselet. Singular optical manipulation of birefringent elastic media using nonsingular beams. Opt. Lett., 34:3229-3231, 2009.

[71] E. Brasselet. Spin-orbit optical cross-phase-modulation. Phys. Rev. A, 82:063836, 2010.

[72] G. F. Calvó and A. Picon. Spin-induced angular momentum switching. Optics Letters, 32(7):838-840, 2007.

[73] E. Karimi, G. Zito, B. Piccirillo, L. Marrucci, and E. Santamato. Hypergeometric-gaussian modes. Opt. Lett., 32(21):3053-3055, 2007.

[74] P. Vaveliuk. Nondiffracting wave properties in radially and azimuthally symmetric optical axis phase plates. Opt. Lett., 34:3641-3643, 2009.

[75] A. M. de M. Carvalho, C. Sátiro, and F. Moraes. Aharonov-bohmlike effect for light propagating in nematics with disclinations. EPL, 80:46002, 2007.

[76] M. J. Padgett and J. Courtial. Poincaré-sphere equivalent for light beams containing orbital angular momentum. Opt. Lett., 24(7):430-432, 1999.

[77] E. Nagali, F. Sciarrino, F. De Martini, B. Piccirillo, E. Karimi, L. Marrucci, and E. Santamato. Polarization control of single photon quantum orbital angular momentum states. Opt. Express, 17(21):18745-18759, 2009.

[78] E. Karimi, S. Slussarenko, B. Piccirillo, L. Marrucci, and E. Santamato. Polarization-controlled evolution of light transverse modes and associated pancharatnam geometric phase in orbital angular momentum. Phys. Rev. A, 81(5), 2010.

[79] B. Coutinho dos Santos, C. E. R. Souza, K. Dechoum, and A. Z. Khoury. Phase conjugation and adiabatic mode conversion in a driven optical parametric oscillator with orbital angular momentum. Phys. Rev. A, 76:053821, 2007.

[80] S. Slussarenko, E. Karimi, B. Piccirillo, L. Marrucci, and E. Santamato. Efficient generation and control of different-order orbital angular momentum states for communication links. J. Opt. Soc. Am. A, 28:61-65, 2011.

[81] S. Slussarenko, E. Karimi, B. Piccirillo, L. Marrucci, and E. Santamato. Universal unitary gate for singlephoton spin-orbit four-dimensional states. Phys. Rev. A, 80(2):022326, 2009.

[82] F. De Martini and F. Sciarrino. Non-linear parametric processes in quantum information. Progress in Quantum Electronics, 29(3-5):165-256, 2005.

[83] G. Molina-Terriza, J. P. Torres, and L. Torner. Twisted photons. Nat. Phys., 3:305-310, 2007.

[84] J. T. Barreiro, N. K. Langford, N. A. Peters, and P. G. Kwiat. Generation of hyperentangled photon pairs.
Phys. Rev. Lett., 95(26):260501, 2005.

[85] J. T. Barreiro, T.-C. Wei, and P. G. Kwiat. Beating the channel capacity limit for linear photonic superdense coding. Nat. Phys., 4(4):282-286, 2008.

[86] L. Aolita and S. P. Walborn. Quantum communication without alignment using multiple-qubit single-photon states. Phys. Rev. Lett., 98(10):100501, 2007.

[87] C. E. R. Souza, C. V. S. Borges, A. Z. Khoury, J. A. O. Huguenin, L. Aolita, and S. P. Walborn. Quantum key distribution without a shared reference frame. Phys. Rev. A, 77:032345, 2008.

[88] L. Marrucci, E. Nagali, F. Sciarrino, L. Sansoni, F. De Martini, B. Piccirillo, E. Karimi, and E. Santamato. Photonic quantum information applications of patterned liquid crystals. Mol. Cryst. Liq. Cryst., 526:108$118,2010$.

[89] C. K. Hong, Z. Y. Ou, and L. Mandel. Measurement of subpicosecond time intervals between two photons by interference. Physical Review Letters, 59(18):2044, 1987.

[90] E. Nagali, L. Sansoni, F. Sciarrino, F. De Martini, L. Marrucci, B. Piccirillo, E. Karimi, and E. Santamato. Optimal quantum cloning of orbital angular momentum photon qubits through hong-ou-mandel coalescence. Nat. Photonics, 3(12):720-723, 2009.

[91] E. Nagali and F. Sciarrino. Generation of hybrid polarization-orbital angular momentum entangled states. Opt. Express, 18(17):18243-18248, 2010.

[92] E. Karimi, J. Leach, S. Slussarenko, B. Piccirillo, L. Marrucci, L. Chen, W. She, S. Franke-Arnold, M. J. Padgett, and E. Santamato. Spin-orbit hybrid entanglement of photons and quantum contextuality. Phys. Rev. A, 82(2):022115, 2010.

[93] A. Mair, A. Vaziri, G. Welhs, and A. Zeilinger. Entanglement of the angular momentum states of photons. Nature, 412(6844):313, 2001.

[94] L. X. Chen and W. L. She. Single-photon spin-orbit entanglement violating a Bell-like inequality. J. Opt. Soc. Am. B, 27:A7-A10, 2010.

[95] C. V. S. Borges, M. Hor-Meyll, J. A. O. Huguenin, and A. Z. Khoury. Bell-like inequality for the spin-orbit separability of a laser beam. Phys. Rev. A, 82:033833, 2010.

[96] L. X. Chen and W. L. She. Teleportation of a controllable orbital angular momentum generator. Phys. Rev. A, 80:063831, 2009.

[97] J. T. Barreiro, T.-C. Wei, and P. G. Kwiat. Remote preparation of single-photon hybrid entangled and vector-polarization states. Phys. Rev. Lett., 105:030407, 2010.

[98] L. X. Chen, , and W. L. She. Hybrid entanglement swapping of photons: Creating the orbital angular momentum bell states and greenberger-horne-zeilinger states. Phys. Rev. A, 83:012306, 2011.

[99] E. Nagali, D. Giovannini, L. Marrucci, S. Slussarenko, E. Santamato, and F. Sciarrino. Experimental optimal cloning of four-dimensional quantum states of photons. Phys. Rev. Lett., 105(7):073602, 2010.

[100] E. V. Moreva, G. A. Maslennikov, S. S. Straupe, and S. P. Kulik. Realization of four-level qudits using biphotons. Phys. Rev. Lett., 97(2):023602, 2006.

[101] Yu. I. Bogdanov, E. V. Moreva, G. A. Maslennikov, R. F. Galeev, S. S. Straupe, and S. P. Kulik. Polarization states of four-dimensional systems based on biphotons. Phys. Rev. A, 73(6):063810, 2006. 
[102] G. Vallone, E. Pomarico, F. De Martini, P. Mataloni, and M. Barbieri. Experimental realization of polarization qutrits from nonmaximally entangled states. Phys. Rev. A, 76(1):012319, 2007.

[103] S.-Y. Baek and Y.-H. Kim. Generating entangled states of two ququarts using linear optical elements. Phys. Rev. A, 75(3):034309, 2007.

[104] E. Nagali, L. Sansoni, L. Marrucci, E. Santamato, and F. Sciarrino. Experimental generation and characterization of single-photon hybrid ququarts based on polarization and orbital angular momentum encoding. Phys. Rev. A, 81:052317, 2010.

[105] D. Bruß, D. P. DiVincenzo, A. Ekert, C. A. Fuchs, C. Macchiavello, and J. A. Smolin. Optimal universal and state-dependent quantum cloning. Phys. Rev. A, 57(4):2368-2378, 1998.

[106] C. E. R. Souza, J. A. O. Huguenin, P. Milman, and A. Z. Khoury. Topological phase for spin-orbit transformations on a laser beam. Phys. Rev. Lett., 99:160401, 2007.

[107] B. Coutinho dos Santos, K. Dechoum, and A. Z. Khoury. Continuous-variable hyperentanglement in a parametric oscillator with orbital angular momentum. Phys. Rev. Lett., 103:230503, 2009.

[108] L. X. Chen and W. L. She. Increasing shannon dimensionality by hyperentanglement of spin and fractional orbital angular momentum. Opt. Lett., 34:1855-1857, 2009.

[109] D. P. Caetano, P. H. Souto Ribeiro, J. T. C. Pardal, and A. Z. Khoury. Quantum image control through polarization entanglement in parametric down-conversion. Phys. Rev. A, 68:023805, 2003.

[110] L. X. Chen and W. L. She. Sorting photons of different rotational doppler shifts (rds) by orbital angular momentum of single-photon with spin-orbit-rds entanglement. Opt. Express, 16:14629-14634, 2008.

[111] P. Zhang, B.-H. Liu, R.-F. Liu, H.-R. Li, F.-L. Li, and G.-C. Guo. Implementation of one-dimensional quantum walks on spin-orbital angular momentum space of photons. Phys. Rev. A, 81:052322, 2010.

[112] C. S. Hamilton, A. Gábris, I. Jex, and S. M. Barnett. Quantum walk with a four-dimensional coin. New J. Phys., 13:013015, 2011.

[113] L. X. Chen and W. L. She. Encoding orbital angular momentum onto multiple spin states based on a huffman tree. New J. Phys., 11:103002, 2009.

[114] C. E. R. Souza and A. Z. Khoury. A michelson controlled-not gate with a single-lens astigmatic mode converter. Opt. Express, 18:9207-9212, 2010.

[115] F. Tamburrini, B. Thide, G. Molina-Terriza and G. Anzolin. Twisting of light around rotating black holes. Nature Phys., 7:195-7, 2011. 